\title{
PQM vs. BPQM: Studying the Tailoring of a General Quality Model to a Specific Domain
}

\author{
Coral Calero $^{1}$, Cristina Cachero ${ }^{2}$, Julio Córdoba ${ }^{2,3}, \mathrm{M}^{\mathrm{a}}$ Ángeles Moraga ${ }^{1}$ \\ ${ }^{1}$ Grupo ALARCOS. University of Castilla-La Mancha (Spain) \\ ${ }^{2}$ Departamento de Lenguajes y Sistemas Informáticos. University of Alicante (Spain) \\ ${ }^{3}$ Centro de Investigacón en Informática Aplicada. Universidad Latina de Costa Rica \\ \{Coral.Calero, MariaAngeles.Moraga@uclm.es\}@uclm.es; $\{$ ccachero,jcordoba\}@dlsi.ua.es
}

\begin{abstract}
In this paper a comparative study carried out between a generic model for portal quality (PQM) and a specific model for eBanking portal quality (BPQM) is presented with the aim to identify the differences and to justify why these have appeared. The goal is to detect gaps in BPQM in order to think about including them in future versions.
\end{abstract}

Keywords: Web portals, e-Banking portals, quality model

\section{Introduction}

Web Portals are emerging Internet-based applications, enabling access to different sources (providers) through a single interface (Mahdavi et al., 2004). They provide personalization, single sign on and content aggregation from different sources, as well as hosting the presentation layer of Information Systems (Java Community Process, 2003). Moreover, they can help users to find the desired information, service or product from among a (large) number of providers effectively, without having to navigate through these one by one (Mahdavi et al., 2004).

The primary objective for the development of portals may vary from one organization to another (Hazra, 2002) although, in general, it can be formulated as 'creating a working environment where users can easily navigate to find the information they specifically need to perform their operational or strategic functions quickly and to make decisions' (Collins, 2001). In order to assure the fulfilment of this objective, certain software quality criteria should be met.

The term "software quality" is used to assess both processes and products. In our case, a product-based view is adopted based on the opinion that the clearer we are about what to achieve in terms of product quality, the easier it will be to tune the process accordingly. This view is also backed by ISO (2001), who stated that evaluating a product can provide feedback for the improvement of a process.

Software product quality is evaluated in accordance with a "quality model". Quality models should consider criteria that satisfy the needs of the developers, 
maintainers, buyers and end users (ISO, 2001). As for their objectives, these models should be used for building better products and to assess and ensure their quality.

Building a quality model is a complex undertaking. Normally, the software product quality is broken down hierarchically into characteristics and sub-characteristics which can be used as a checklist. However, trying to define a quality model that fits any software product regardless of the differences between it and other types is not sufficient. Faced with this fact, two approaches are possible: either to define a new quality model from scratch, or to tailor any of the existing models (e.g. the ISO 9126 quality model) to fit specific domains (ISO, 2001).

Whatever the case, if developing a quality-assured Web portal is important for those organizations aiming to provide services through the Web, this fact is especially relevant for eBanking Web portals. The reason is threefold:

- Firstly, as a banking channel, the Internet has certainly experienced strong growth in customer uptake in recent years, with predicted European compound annual growth rates of approximately $9 \%$; that may be up to $15 \%$ in countries like France [Reuters Report]. This banking channel competes in importance with actual physical branches and call centres. The importance of this means may grow if we focus on simple transactions (e.g. making payments, transferring funds and checking balances). In fact, according to a recent survey (Córdoba el al., 2007) the frequency of use of eBanking solutions among bank clients oscillates between daily and weekly, a rate that is similar to that of Automatic Teller Machines (ATMs). Indeed, banks are the driving force in the economic development of countries, as they are the support for the importation and exportation of most products and services.

- Secondly, banks acquired a competitive advantage by being the first to market with Internet banking services before there was widespread availability of these. This fact served as a useful marketing tool to attract more technologically-aware consumers. Now, however, when Internet banking services are virtually omnipresent, a bank's promise of access to these services is no longer enough to woo consumers. Instead, banks are forced to give their services some features that make them different, by using quality "hooks", such as enhanced functionalities and effective Web design. If they don't do this, there is a risk that technologicallyaware consumers who chose a bank in the first place because of its eBanking solution, will now switch to another because they have found an even better product.

- Thirdly, certain bank quality characteristics are bound up with the core of the business itself. For example, the conditions under which some kinds of operations are performed are regulated by tight requirements and governmental laws, so the developers must be sure that they are enforced in the application.

BPQM is our attempt to meet all these needs. This model used expert consultation to extract which of the general portal quality characteristics, gathered mainly from PQM (a portal quality model), were relevant for the eBanking domain. Next, the model was completed with a set of new characteristics/sub-characteristics, not present in any of the sources. Finally, the quality model was validated through a survey performed on 168 eBanking users. In this paper, we present the comparative study carried out between PQM and BPQM models to identify the differences and to justify 
why these have appeared. The goal is to detect gaps in the model, in order to think about including them in future versions of BPQM.

Sections two and three provide an introduction to the quality models, PQM and BPQM. Section 4 presents the comparative study and the conclusions obtained from those. Finally, the overall conclusions and proposed future work can be found in the last section.

\section{PQM: the Portal Quality Model}

In (Moraga et al., 2006), a generic quality model for portals, namely PQM (Portal Quality Model) was put forward and compared to other portal quality model proposals. After the comparison, PQM stood out as being the most complete and this is the reason why we used it as basis for the creation of BPQM. This model was made using the SERVQUAL model proposed by (Parasuraman et al., 1998) as the basis, along with the GQM (Goal Question Metric) method (Basili et al., 1994). The characteristics identified for the PQM model are:

- Tangible: characteristic of the portal that indicates whether it contains all the software and hardware infrastructures needed, according to its functionality. The sub-characteristics are:

- Adaptability. Ability of the portal to be adapted to different devices (for instance PDA's, PCs, mobile phones, etc).

- Transparent access. Ability of the portal to provide access to the resources, while at the same time isolating the user from their complexity.

- Reliability: ability of the portal to perform its functionality accurately. In addition, this characteristic will be affected by:

- Fault tolerance. Capability of the portal to maintain a specified level of performance in the event of software faults (for example, a fault during the sending of information or the execution of a job).

- Resource utilization. Capability of the portal to offer its resources to the user according to his profile or particular role or privileges.

- Availability. Capability of the portal to be always operative, so that users may be able to access it and use it anywhere, anytime.

- Search Quality. Appropriateness of the results that the portal provides when undertaking a search/request made by the user.

- Responsiveness: willingness of the portal to help and provide its functionality in an immediate form to the users. In this characteristic, the sub-characteristics are:

- Scalability. Ability of the portal to adapt smoothly to increasing workloads which come about as the result of additional users, an increase in traffic volume, or the execution of more complex transactions.

- Speed. Ability of the portal to remain within the response time boundaries tolerated by portal users.

- Empathy: ability of the portal to provide caring and individual attention. This dimension has the following sub-characteristics:

- Navigation: Simplicity and intuitiveness of the navigation paths provided by the portal. 
- Presentation: Clarity and uniformity of the interface.

- Integration: Degree of global portal coherence achieved after the inclusion of the components that make up the portal. All the components of the portal must be integrated in a coherent form.

- Personalization: The portal's capability to adapt to the user's priorities.

- Data quality (DQ): This characteristic is defined as the quality of the data contained in the portal. It has four sub-characteristics:

- Intrinsic $D Q$. Degree of care taken in the creation and preparation of information.

- Representation $D Q$. Degree of care taken in the presentation and organization of information for users.

- Accessibility $D Q$. Degree of freedom that users have to use data, define and/or refine the manner in which information is input, processed or presented to them.

- Contextual DQ. Degree to which the information provided meets the needs of the users.

- Security: capability of the portal to prevent, reduce, and respond to malicious attacks adequately. Its sub-characteristics are:

- Access control. Capability of the portal to allow access to its resources only to authorized people. Thus, the portal must be able to identify, authenticate, and authorize its users.

- Security control. Capability of the portal to carry out auditing of security and to detect attacks. The auditing of security shows the degree to which security personnel are enabled to audit the status and use of security mechanisms by analyzing security-related events. In addition, attack detection seeks to detect, record and notify attempted attacks as well as successful attacks.

- Confidentiality: Ability to guard the privacy of the users.

- Integrity. Capability of the portal to protect components (of data, hardware and software) from intentional or unauthorized modifications.

\section{BPQM: The eBanking Portal Quality Model}

The purpose of creating the BPQM has been the integration of relevant characteristics for eBanking that are present in PQM, completing them by filling in the gaps detected in a survey performed among domain experts.

These gaps were filled in through the validation of the model, performed using an "eBanking User Satisfaction Survey" that was carried out during the months of February and April 2007 with the aim of evaluating satisfaction levels based on Internet banking user experience. In the production of the survey, the suggestions proposed in (Pfleeger and Kitchenham, 2006) were taken into account. The survey was printed and physically distributed to 200 people randomly selected from the eBanking user community of Costa Rica. From the total number of individual surveys given out, 22 were left in blank, 10 were partially completed, and 168 were fully answered, which gives a result of an $84 \%$ response index. For the validation of the quality model, these last 168 results were considered. 
This work generated the following list of characteristics:

- Empathy: capacity of the portal to provide personalised help and attention. Within this characteristic we would highlight:

- Navigability: capacity of the portal to offer users an intuitive and easy-to-use navigation system.

- Personalisation: capacity of the portal to allow users to customise their services so that user effort is reduced and client satisfaction is increased.

- Usability: capacity of a software product to be understood, learnt and used, as well as to be attractive, always bearing in mind regulations and usability guides. Within this characteristic the following sub-characteristics are found:

- Degree of attractiveness. The portal must be able to satisfy the wishes of users, not only in terms of visual appearance, but also through its services and the degree of satisfaction achieved in its deployment.

- Accessibility. This refers to the freedom of users when using data, by defining and/or refining the manner by which the information is entered, processed or presented to them.

- Learning capacity. It focuses on the amount of effort needed by the user to learn how to use services and functionalities that are available in the portal.

- Efficiency: capacity of a software product to provide an appropriate performance which takes into account the amount of resources used and which is adaptable to specific conditions. This characteristic includes:

- Response time: It focuses on the response times after a user request. There are various important response times for a banking portal: the time that elapses between when the solution indicates to the user that it is processing a request (state bar or progress bar) and the time taken by the solution in delivering a final response, whether positive or negative, the latter through an error message).

- Security: It represents the "capacity of the software product to avoid unauthorised access, either accidental or deliberate, to programs and data". For this characteristic we point out:

- Integrity: The portal must protect the data and information in such a way that no deliberate or unauthorised modifications take place.

- Confidentiality: The main focus is on preserving the privacy of users.

- Fault tolerance: It refers to the capacity of offering a clear response to the user when faults, errors, or attacks occur. The portal must, at all times, recover in the shortest period of time and affect user services as little as possible.

- Functionality: It refers to the "capacity of a software product to satisfy the established functional requirements and the implicit needs of users". It includes:

- Maintainability: The portal must be built in such a way that it facilitates the easy and rapid solution of any type of error, as well as the maintenance and updating of information.

- Interoperability: It focuses on the ability of the portal to interact with other systems and services. 
- Available services: The portal must have the maximum amount of services, which should be available 24 hours a day.

\section{PQM vs. BPQM}

Having given an overview of both models, this section presents the set of similarities and differences that have been detected between them. Possible reasons have been found to explain why, with respect to some of the discrepancies, those characteristics have not been identified either by experts or by the eBanking users we interviewed as being relevant for eBanking portals. For other differences, however, no explanation could be found, which in turn suggests a need for further empirical research to decide about their incorporation into the BPQM model.

Table 1 shows the comparison made between both models. Black cells mean equality in the sub characteristic in both models. Dark grey cells mean some kind of similarity (partial coverage of a sub characteristic, same meaning but different name, etc) while rows or columns in grey imply that there is not any kind of coincidence. In the next sub-sections, the results of the study are explained.

\subsection{Sub-characteristics in PQM and in BPQM}

As shown in Table 1 above, there are nine sub-characteristics that are common to both models: (1) Fault tolerance, (2) Availability, (3) Navigation, (4) Personalization, (5) Confidentiality, (6) Integrity, (7) Accessibility DQ, (8) Access control and (9) Speed.

Most of these characteristics are mainly related to the comfort of the user when using the portal $(2,3,4,7,9)$ and to security aspects, which are very relevant when the portal is used as part of the user activities $(1,56,8)$. These aspects coincide with the definition of a portal given by Collins (2001): "a working environment where users can easily navigate to find the information they specifically need to make decisions and to perform their operational or strategic functions quickly". We would like to point out how our survey showed that, for $68 \%$ of the interviewees, a $24 / 7$ service availability is absolutely essential if user objectives are to be fulfilled. Whether the weight of this characteristic is similarly important in other kinds of portals remains an open issue and this will be investigated.

It must be noted that BPQM considers access control as an integral part of confidentiality and that personalization includes both static and dynamic techniques.

\subsection{Sub-characteristics in PQM and partially in BPQM}

There are four sub-characteristics of PQM that are partially supported in BPQM: (1) Presentation (with attractiveness), (2) Integration (with attractiveness) , (3) Transparent access (with learnability) and (4) Scalability (with response time and fault tolerance).

Learnability (3) is a characteristic that, although not included in PQM, plays a dominant role in eBanking applications. This is because the Internet does not make 
personal interaction with a bank employee possible and to input information online can be difficult. For this reason, banks consider the Internet to be a poor channel for advice and sales. In fact, their branches continue to generate more business for banks than other means and the number of branches has therefore not decreased significantly, despite their being the most expensive to maintain (Reuters, 2004). Improving help mechanisms and guiding the customer throughout online interaction is a necessary step in increasing clients' confidence in Internet so that they see it as a convenient point of sale (POS) for them to access high-value, complex products. These kind of products are those where customers still require the reassurance of speaking to a bank advisor.

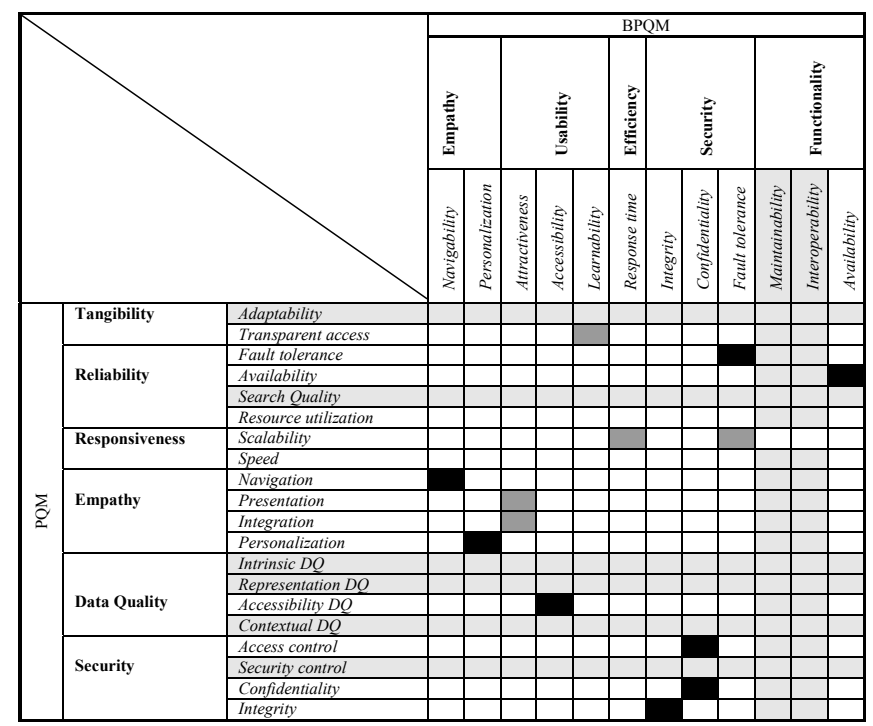

Table 1. PQM sub-characteristics vs. BPQM sub-characteristics

Given that (1) bank customers are not Internet experts and that (2) eBanking applications are a service channel, there is a need to educate and train clients in the use of the Internet as a means or channel of access to banking services. The survey demonstrated that the incorporation of services such as online help, tutorials or simulations increases user satisfaction. According to Hudson (2007) and Centeno (2003) training is needed to improve the penetration, acceptance, and satisfaction of banking portal users. We believe that learnability (BQPM) is related to transparent access (PQM) since both aim to reduce application complexity for the user.

BPQM considers that attractiveness involves, though is not limited to, presentation and integration of the application $(1,2)$.

Lastly, BPQM points out that scalability (4) is covered by response time and fault tolerance. The reason is that an eBanking customer is not interested in how many accesses the application can support at the same time, but rather in whether his requests are carried out quickly and without errors. 


\subsection{Sub-characteristics in PQM and not in BPQM}

There are seven characteristics in PQM that have been not considered in BPQM: (1) Adaptability, (2) Resource utilization, (3) Intrinsic DQ, (4) Representational DQ, (5) Contextual DQ, (6) Search quality and (7) Security control.

PQM includes a whole section devoted to quality of data $(3,4,5)$ which, in the case of BPQM, is not covered completely. The reason is that most data are very specific and/or directly come from databases (e.g. account states) or external sources (e.g. stock prices) and so authoring of content was not considered to be a significant activity during eBanking portal development. Nevertheless, the survey showed that data-intensive help mechanisms are necessary and would be a candidate for its inclusion in the BPQM model.

As far as adaptability and resource utilization $(1,2)$ is concerned, so far we have not found interest on this topic among the users interviewed. The lack of concerns about adaptability (support for different devices) might be due to the fact that some channels, such as mobile devices, have not been considered important. We think that this may change in the future, however, so that banks should start getting ready to meet this need.

From the user point of view, the background to the e-banking solution is not something she knows or cares about; the user just knows that the application is very slow or that it fails. In any case, it would be necessary to work more on these important aspects in order to determine their inclusion in BPQM.

It seems logical that users have not said that search quality (6) is an important subcharacteristic of BPQM, since searching is not a prime functionality of this kind of portals. Basically, the user expects to find in the portal the same kind of services as are available in a branch. It is not normal for users to look for information, much less information requiring search tools. However, this aspect must be studied in depth in order to decide whether it must be added to the model or not, due to the increasing diversification of services that banks are starting to offer.

Finally, in this section, the security control (7) is the most suspicious result from the ones obtained in this section. eBanking users do not seem to be interested in how the bank monitors attacks and audits the status and use of security mechanisms, as long as their data remain confidential and show integrity. We do not expect such a situation to change in the near future, so we have dismissed this characteristic as being relevant for BPQM.

\subsection{Sub-characteristics not in PQM and in BPQM}

Finally, there are two sub-characteristics included in BPQM but not considered in PQM: (1) Maintainability and (2) Interoperability.

Users expect eBanking applications to have a high maintainability. This means that they expect the bank to maintain the accuracy of the data provided and to respond rapidly to any problems encountered to any lack of functionality detected during use (1). In addition, users require the eBanking portals to provide the possibility of interconnecting with different applications. Since a bank is a financial operator, the interoperability (2) with third parties is vital for users. In the survey, $90 \%$ of eBanking 
users said they were not satisfied with the number of third parties that could interoperate with their bank.

\subsection{Conclusions of the study}

As result of the study, we obtained that the BPQM includes $45 \%$ of PQM subcharacteristics completely and that $20 \%$ of the PQM sub-characteristics are also considered, although not completely. The remaining sub-characteristics are not considered in the BPQM for the time being. We believe that all of the characteristics, with the exception of security control, should be considered for inclusion in BPQM, as they could become quality indicators to evaluate how well the eBanking application has foreseen changes in the way bank service types and user access devices may evolve in the future. Finally, by means of the survey, two subcharacteristics excluded in PQM have been identified as important for BPQM.

Conversely, we need to further give consideration to whether scalability and security control should be regarded as characteristics of PQM. On one hand, our survey has shown that from a user perspective, scalability is related to fault tolerance and speed. Both of these characteristics are also included in PQM. Regarding security control, it seems that users are not interested in how security personnel audit information as long as the control is confidential and shows integrity ( these characteristics are also present in PQM).

\section{Conclusions and future work}

Nowadays, eBanking solutions are strengthening their position as the main channels for banking operations. This makes us conclude that the quality of these channels will be fundamental for the survival of banks in the global economy. Furthermore, banks have seen the need to transform their eBanking solutions from simple transactions to systems that provide integrated services similar to those available in an actual bank office (with customer service and support).

In an initial work on this (Córdoba et al., 2007), and basing ourselves on PQM, we tried to define a quality model for bank portals. This work included a 168-user survey, designed to determine the characteristics and sub-characteristics of the new model. However, since the creation process of a model is not a trivial issue (even when it may be based on a generic model), the objective was to know whether the model defined was correct or rather inaccurate. It might have left something out (due to possible subjectivity of users in the survey participation).

To this end, we have carried out a comparison between both models, concluding that BPQM includes $45 \%$ of PQM sub-characteristics completely, while $20 \%$ of the PQM sub-characteristics are also considered, although not completely. The remaining subcharacteristics are not considered in BPQM for now. Although it is logical for some of these not to be included in the model, others need to be studied in depth in order to determine if they must be components of the final model or not.

Lastly, two sub-characteristics not included in PQM have been identified in the survey as being important for BPQM. 
For future work, it would be important to validate the current characteristics, with new users and the new characteristics. Once the model is finalised, and with the purpose of making it applicable in practice, we would aim to define measures for each sub-characteristic of the model so that it can be applied to different eBanking solutions by different people, attempting to generate a quality comparison (ranking) between the banks within a specific region (i.e.: Costa Rica, Spain, etc.).

\section{Acknowledgements}

This paper has been supported by the MEC Spanish Ministry projects CALIPSO (TIN20005-24055-E), MEC-FEDER (TIN2004-03145), ESFINGE (TIN2006-15175C05-05), METASIGN (TIN2004-00779) and DSDM (TIN2005-25866-E)). Also, it is part of the DADASMECA project (GV05/220), financed by the Valencia Government and DIMENSIONS (PBC-05-012-1) financed by the Castilla-La Mancha Government.

\section{References}

Basili, V. R., C. Caldiera, et al. (1994). Goal Question Metric Paradigm. Encyclopedia of Software Engineering. Vol. 1, John Wiley \& Sons.

Centeno, C. Adoption of Internet Services in the Enlarged European Union. Lessons from the Internet banking case. Institute for Prospective Technological Studies, European Commission, Joint Research Centre, Report EUR 20822 EN. June 2003.

Collins, H. (2001). Corporate Portals, New York, Amacom

Córdoba, J., Cachero, C., Calero, C., Genero, M. and Marhuenda, y. (2007). Modelo de calidad para portales bancarios. Sent to CLEI 2007. In Spanish

Hazra, T. K. (2002). Building Enterprise portals: principles to practice. 24 International Conference on Software Engineering., pp. 623-633.

Hudson, W. The Lost World of E-Banking. SIGCHI Bulletin September/October 2002, Syntagm Ltd USA. [Abr. 14, 2007], available on the Internet: http://www.syntagm.co.uk/design/articles/ebanking.htm

ISO/IEC TR 9126-1. Software engineering - Product quality - Part 1: Quality model

Mahdavi, M., J. Shepherd, and B. Benatallah. A Collaborative Approach for Caching Dynamic Data in Portal Applications. 2004. Proceedings of the fifteenth conference on Australian database Vol.27.pp. 181-188.

Moraga, M., Calero, C. and Piattini, M. (2006) Comparing different quality models. Online Information Review Journal.

Parasuraman, A., V. A. Zeithami, et al. (1998). SERVQUAL: a multi-item scale for measuring consumer perceptions of service quality. Journal of Retailing. Vol. 67(4) pp. 420-450.

Pfleeger, S y Kitchenham, B. Principles of Survey Research, Part1-Part6. Software Engineering Notes 26(6), 27(1), 27(2), 27(3), 27(5), 28(2). ACM SIFSOFT. 2001 - 2003.

Reuters (2004). The European eBanking and ePayments market outlook. Reuters-Business insights. August 2004. MBA Group Limited, 